\title{
Partial Structure Factors of Polyisoprene: Neutron Scattering and Molecular Dynamics Simulation
}

\section{F. Alvarez, ${ }^{\dagger}$ J . Colmenero, ${ }^{*, t, \pm}$ R. Zorn, ${ }^{\S}$ L. Willner,,$^{\S}$ and D. Richter ${ }^{\S}$}

Departamento de Física de Materiales, Universidad del País Vasco (UPV/ EHU) y Unidad de Física de Materiales (CSIC-UPV/ EHU), Apartado 1072, 20080 San Sebastián, Spain, Donostia International Physics Center, Paseo Manuel de Lardizabal 4, 20018 San Sebastián, Spain, and Institut für

Festkörperforschung, Forschungszentrum J ülich, D-52425, J ülich, Germany

Received August 28, 2002; Revised Manuscript Received October 28, 2002

\begin{abstract}
In this paper, we have combined molecular dynamics simulation and neutron diffraction experiments with polarization analysis, to unravel the different atomic correlations contributing to the total and partial static structure factors of polyisoprene (PI). Four different PI samples have been investigated: PId3 (methyl group deuterated and main chain protonated); PI d5 (methyl group protonated and main chain deuterated); PId8 (fully deuterated); PIh8 (fully protonated). The neutron diffraction experiments with polarization analysis were carried out by means of the diffuse scattering spectrometer D7 at the Institute Laue Langevin (ILL, Grenoble, France). By means of this technique the partial static structure factors corresponding to the PI h8, PId3, and PI d5 samples and the total static structure factor $\mathrm{S}(\mathrm{Q})$ (PId8) were obtained in absolute units in the wavenumber regime $\mathrm{Q} \leq 4 \AA^{-1}$. In addition, the temperature evolution of $S(Q)$ was al so measured by a neutron powder diffractometer (D20, ILL) without polarization analysis but in a wider $\mathrm{Q}$ range $\mathrm{Q} \leq 13 \AA^{-1}$. On the other hand, fully atomistic molecular dynamic (MD) simulations were carried out at different temperatures on a model of PI built by means of the amor phous-cell protocole. The static structure factors measured on the different samples were also calculated from the simulation data. The agreement found between simulation and measurements shows that our simulation cell is a realistic representation of the actual structure of PI. Taking advantage of the information contained in the simulation runs, we have unambiguously identified the different atomic correlations contributing to the different "peaks" of the total and partial structure factors measured. In particular, we have shown that a "prepeak" present in some of the data is not related to intermediate range order but is naturally explained by the interplay of the different partial structure factors, a result which may have some bearing al so for other systems. In addition, we have found-both by experimental and by simulations - that the intensity of the first intermol ecular peak of the total static structure factor $\mathrm{S}(\mathrm{Q})$ strongly increases with temperature. Although a full understanding of this phenomenon will need further work, we have been able to identify the main atomic correlations involved in this temperature evolution.
\end{abstract}

\section{Introduction}

Although nowadays it is generally accepted that the conformation of polymer chains in the bulk follows the random coil model proposed by Flory, ${ }^{1}$ the short-range order of polymer melts and glassy polymers is still poorly understood. The effects of short-range order become apparent in a diffraction experiment at $Q$ values (Q: wavenumber) higher than about $0.5 \AA^{-1}$. A typical diffraction pattern obtained, for example, by neutron scattering (NS) from a fully deuterated polymer shows marked diffraction maxima at $Q$ values between 1 and $2 \AA^{-1}$ which, in principle, correspond to interchain correlations and weaker maxima at higher values, corresponding to intrachain correlations. ${ }^{2}$ However, almost nothing is known about the relationship between these maxima and the particular chemical structure of the monomer or the microstructure of the chain. For instance, some polymers as 1,4-polybutadiene or poly(vinyl chloride) show only one "intermolecular" peak

* Corresponding author: Telelephone: +34 943015962 . Fax: +34943 015600. E-mail: wapcolej@sc.ehu.es.

† Universidad del País Vasco (UPV/EHU) y Unidad de Física de Materiales (CSIC-UPV/EHU).

‡ Donostia International Physics Center.

$\S$ F orschungszentrum J ülich. while others display a peak with a shoulder or even two clearly distinguished peaks as, e.g., polyisoprene. ${ }^{3}$ It is noteworthy that the presence of such shoulders or second peaks in the intermolecular range of $\mathrm{Q}$ is not always correlated with the existence of a bulky side group in the monomer, as could be expected from simple arguments.

For an amorphous system the structural information is contained in the so-called radial distribution function $g(r)$ or in its Fourier transform counterpart: the static structure factor $\mathrm{S}(\mathrm{Q})$. In the case of amorphous polymers composed by carbons and hydrogens, S(Q) can be measured by neutron diffraction using a fully deuterated sample, i.e., a sample where all hydrogens are replaced by deuterons. As the neutron scattering lengths of carbon and deuteron are very si milar, both atoms are al most indistinguishable from neutrons. However, in the case of complicated mol ecular systems as polymers, the average structural information contained in either $\mathrm{g}(\mathrm{r})$ or $\mathrm{S}(\mathrm{Q})$ does not allow to easily unveil the short range order details. It is therefore of utmost importance in these cases to obtain additional information on the particular correlations arising from groups of selected atoms. This knowledge can be achieved by the study of partial static structure factors or partial radial distribu- 
tion functions. For instance, in the case of polymers only composed by carbon and hydrogen atoms, the X-ray diffraction intensity mainly highlights the carboncarbon correlations due to the values of the X-ray scattering factors of carbon and hydrogen. Experimentally, different partial structure factors can be accessed by means of the isotopic substitution techniques combined with neutron diffraction with polarization analysis. In the case of polymers, the isotopic substitution techniques are based on the very different neutron scattering lengths of hydrogen and deuteron atoms (see section 3). Moreover, it seems that substitution of hydrogen by deuteron atoms hardly affects the structural properties of amorphous polymers in general. Thus, by taking advantage of these features, one can profit of selective deuteration labeling techniques in order to obtain the different partial contributions to the total static structure factor arising from differently deuterated samples. On the other hand, neutron diffraction measurements with polarization analysis allows separating the coherent and incoherent contributions to the scattering and provides coherent cross section at an absolute scale. This kind of measurements can be carried out by means of the so-called diffuse scattering spectrometers as, for instance, the D7 instrument at the Institute Laue-Langevin (ILL) in Grenoble, France. However, we have to point out that, though these techniques access experimentally the partial static structure factors in absolute scales, the interpretation of the results in terms of the atomic correlations is usually extremely difficult.

On the other hand, modern computer simulation techniques seem to be one of the most promising tools for unraveling the local structure and the dynamics of polymer melts. First of all, computer simulation enables precise insight into the atomic structure and dynamics. Second, one can artificially modify in an easy way the scattering lengths of partial groups of atoms in the simulation, thus enhancing particular correlations in the calculated structure factors. However, to do this, fully atomistic and well-equilibrated samples are necessary, i.e., first of all the simulated cells have to be validated by extensive scattering experiments. After this comparison, the simulation conditions can also be improved. Thereby, it seems that a combination of computer simulation and neutron diffraction experiments with polarization analysis on partially deuterated samples, is by now the best route to provide new insight into the problem of the short-range order in polymers. To our knowledge, a coordinated effort in this line has only been carried out in the case of polycarbonate samples. ${ }^{4}$ In this work we have followed a similar scheme for a simpler but very important polymer: polyisoprene (PI). In this case, recent molecular dynamics (MD) simulations results ${ }^{5-8}$ indicate that realistic samples can be simulated without using the "inverse mapping" technique which was applied in the case of polycarbonates. Four different PI samples are investigated: PId3 (methyl group deuterated and main chain protonated); PId5 (methyl group protonated and main chain deuterated); PI d8 (fully deuterated); PIh8 (fully protonated) in a wide $\mathrm{Q}$ range covering the inter- and intramacromolecular range.

After this introduction, the paper is organized as follows. Sections 2 and 3 describe the experimental and MD-simulation methods in detail. Then, the results from both approaches will be presented and compared in section 4. The interpretation of the different static structure factors measured and calculated, in terms of atomic correlations, will be presented in the discussion section 5 . Finally, section 6 summarizes the main results and conclusions.

\section{Samples and Experimental Method}

a. Synthesis and Characterization of Polyisoprenes. The polyisoprenes $\left[-\mathrm{CH}_{2}-\mathrm{CH}=\mathrm{C}\left(\mathrm{CH}_{3}\right)-\mathrm{CH}_{2}-\right]_{\mathrm{n}}$ used in this study were prepared by anionic polymerization in benzene with sec-butyllithium as initiator and methanol as termination reagent. The deuterated monomers, isoprene- $d_{8}$, isoprene- $h_{5}-$ methyl- $_{3}$, and isoprene$d_{5}$-methyl- $h_{3}$, were purchased from Cambridge I sotope Labaratories, Andover, MA. The polymerizations were carried out under high vacuum in sealed glass reactors. The techniques applied and the protocols for purification of reagents were similar to those specified in the literature.9,10 After termination the polymers were isolated by precipitation in methanol and then dried in a vacuum at $40{ }^{\circ} \mathrm{C}$ for several days in order to remove all volatile in particular hydrogen-containing impurities.

The polymers were characterized by size exclusion chromatography using a Waters $150 \mathrm{C}$ gel permeations chromatograph. For separation, a set of four columns was utilized covering a porosity range of 100 to $4 \times 10^{6}$ $\AA$. The columns were calibrated by a set of narrow molecular weight polystyrenes. Tetrahydrofuran was the elution solvent at a flux of $1 \mathrm{~mL} / \mathrm{min}$. All chromatograms exhibit a sharp signal revealing small polydispersities for the polymerization products. In the case of PId5, a second small peak ( $2 \%$ area) was observed in the spectrum at an elution time that corresponds to a doubled molecular weight. Coupled material was observed also in other polymerization products of the $d_{5} h_{3}$-monomer. Apparently it relates to an impurity that cannot be removed by standard purification processes. However, the presence of the high molecular weight impurity is not expected to significantly influence the results of the present study. Therefore, the material was used as obtained.

The number-average molecular weights were determined by membrane osmometry. The measurements of the osmotic pressure $\pi$ were performed with a Knauer instrument at $37^{\circ} \mathrm{C}$ in toluene. The molecular weights were extracted from $(\pi / \mathrm{c})^{1 / 2}$ vs $\mathrm{c}$ plots, where $\mathrm{c}$ is the polymer concentration.

The h-content of the partially deuterated and the perdeuterated polyisoprenes were determined by ${ }^{1} \mathrm{H}$ NMR measurements. 1,1,2,2-Tetrabromoethane was taken as internal reference. In the spectra either the signals of the methyl groups $\left(d_{3} h_{5}\right)$ or the signals due to the backbone $\left(d_{5} h_{3}\right)$ or all resonances $\left(d_{8}\right)$ almost disappeared. The integrals of the residual signals together with those of the other protons were related to the integral of the reference. Thus, the average number of protons per repeat unit could be calculated. The important characteristics of the polyisoprene samples are summarized in Table 1.

b. Experimental Techniques and Method. (1) D20 Measurements. D20 (I LL, Grenoble, France) is a high-flux, medium resolution powder diffractometer equipped with a large-area linear curved positionsensitive detector. The incident wavelength was set to $0.88 \AA$ enabling a range of scattering vectors $Q$ up to 
Table 1. Characteristics of Polyisoprene Samples

\begin{tabular}{clcc}
\hline polymer & $\mathrm{H} / \mathrm{ru}^{\mathrm{a}}$ & $\mathrm{M}_{\mathrm{n}} / 10^{5 \mathrm{~b}}$ & $\mathrm{M}_{\mathrm{w}} / \mathrm{M}_{\mathrm{n}}{ }^{\mathrm{c}}$ \\
\hline PIh8 & 8 & 1.06 & 1.04 \\
PId3 & 5.16 & 1.08 & 1.03 \\
PId5 & 3.11 & 1.23 & 1.03 \\
PId8 & 0.155 & 1.24 & 1.03
\end{tabular}

a Protons per repeat unit, determined by ${ }^{1} \mathrm{H}$ NMR. ${ }^{b}$ Membrane osmometry. ' Size exclusion chromatography.

$13.8 \AA^{-1}$. Because D20 has no option of separating incoherent and coherent scattering, only the fully deuterated (mostly coherently scattering) sample was measured. A film of about $0.55 \mathrm{~mm}$ thickness was pressed between vanadium foils and put as a hollow cylinder into a cylindrical vanadium sample holder of $15 \mathrm{~mm}$ diameter. The use of vanadium was imperative because it is the only sample holder material showing nearly no Bragg peaks over the large $Q$ range. The sample was measured at temperatures $2,100,139,178,219,269$, and $314 \mathrm{~K}$ in a helium cryostat. The primary data treatment consisted of a detector efficiency correction using a vanadium standard and angular calibration. The correction for background scattering was done by empty cell and cadmium measurements. After these standard corrections, the intensity measured $I_{\exp }(\mathrm{Q})$ at all temperatures shows a systematic decrease with Q. This well-known tendency is related to inelasticity effects, ${ }^{11,12}$ which cannot be easily evaluated for the instrumental conditions, in particular, for polymer samples (see also "D7 measurements" below). Different empirical methods of correction have been proposed, which usually take into account a polynomial $\mathrm{Q}$ dependence of the incoherent contribution to the intensity measured (see, e.g., ref 13). Here we have used a simple correction factor linear in $Q: N(Q)=c_{1}+c_{2} Q$. The values of $C_{1}$ and $c_{2}$ parameters were chosen, first of all, to give the correct high-Q limit of $I(Q)=N(Q) I_{\text {exp }}(Q)$, which only depends on the scattering cross sections of the sample. Moreover, in the $\mathrm{Q}$ range of the first maximum of $I_{\exp }(Q)\left(\sim 1.3 \AA^{-1}\right)$, where the inelasticity effects are less important, the chosen $N(Q)$ also gives the actual value of I(Q)-in absolute units-as it is deduced from D7 measurements on the same sample (see below).

(2) D7 Measurements. D7 (ILL, Grenoble, France) is a diffuse scattering spectrometer capable of 3-directional polarization analysis. In our experiments only the $z$ direction analysis was used in order to discriminate coherent and incoherent scattering. Also the time-offlight option was not used; i.e., we did not perform energy analysis on the scattered neutrons. The incident wavelength was set to $3.02 \AA$ enabling a range of scattering vectors $Q$ up to $4.16 \AA^{-1}$. The samples were put as films into a hollow-cylindrical al uminum sample holder of $30 \mathrm{~mm}$ diameter. The transmissions of the samples were in the range $0.8-0.9$ corresponding to film thicknesses of $0.1-0.4 \mathrm{~mm}$ for the different PI species. Each sample was measured at 2 and $100 \mathrm{~K}$. The correction for background scattering was done by empty cell and cadmium measurements. A vanadium standard was measured as an alternative to the intrinsic incoherent scattering calibration (see below).

If the incoherent scattering arises solely from spin disorder, it flips the neutron spin (down) with probability $2 / 3$ while coherent scattering leaves the spin unchanged (up). From the ratio of the numbers of neutrons scattered with both spin orientations the differential coherent scattering cross-section can be calculated by

$$
\frac{\mathrm{d} \sigma_{\mathrm{coh}}}{\mathrm{d} \Omega}(\mathrm{Q})=\frac{2}{3} \frac{\sigma_{\mathrm{inc}}}{4 \pi}\left(\frac{\mathrm{n}_{\mathrm{up}}}{\mathrm{n}_{\mathrm{down}}}(\mathrm{Q})-\frac{1}{2}\right)
$$

Because all instrument and sample dependent quantities (amount of sample in the beam, primary beam intensity, detector efficiencies etc.) cancel out in the derivation of this formula, only the knowledge of the (tabulated) incoherent cross-section is necessary (Table 2). In this way the uncertainty connected with the usual "vanadium calibration" can be avoided.

Under realistic conditions several corrections have to be applied to the value obtained by the straightforward expression 1 (their effects are shown in Figure la for a representative example):

(1) First, multiple scattering has to be taken into account. This is a rather complicated problem for neutron scattering with polarization analysis because consecutive flipping of the neutron spin might restore its original direction leading to an "apparent coherent scattering". An analytical method has been developed to cope with this situation. ${ }^{14}$ The assumptions used in its derivation are (i) subsequent scattering events have the same relative probability and (ii) multiple scattering is isotropic (also coherent-coherent scattering). This leads to a linear relation between the corrected and uncorrected differential cross sections

$$
\frac{\mathrm{d} \sigma_{\mathrm{coh}}}{\mathrm{d} \Omega}=\mathrm{A}\left[\frac{\mathrm{d} \sigma_{\text {coh }}}{\mathrm{d} \Omega}\right]_{\text {single }}-\mathrm{B}
$$

with the correction coefficients

$$
\begin{aligned}
& \mathrm{A}=\frac{3\left(\sigma_{\mathrm{inc}}+\overline{\sigma_{\mathrm{coh}}}+\sigma_{\mathrm{abs}}\right)^{2}}{\left(\left(4-\mathrm{T}^{\prime}\right) \sigma_{\mathrm{inc}}+3 \sigma_{\mathrm{abs}}+3 \mathrm{~T}^{\prime} \overline{\sigma_{\mathrm{coh}}}\right)\left(\sigma_{\mathrm{abs}}+\mathrm{T}^{\prime}\left(\sigma_{\mathrm{inc}}+\overline{\sigma_{\mathrm{coh}}}\right)\right)} \\
& B=\left(1-T^{\prime}\right) \times \\
& \frac{3 \mathrm{~T}^{\prime}{\overline{\sigma_{\mathrm{coh}}}}^{3}+\left(2 \mathrm{~T}^{\prime}+1\right) \sigma_{\mathrm{inc}}{\overline{\sigma_{\mathrm{coh}}}}^{2}+\left(2-\mathrm{T}^{\prime}\right) \sigma_{\mathrm{inc}}{ }^{2} \overline{\sigma_{\mathrm{coh}}}+\sigma_{\mathrm{inc}}{ }^{3}+\left(\sigma_{\mathrm{inc}}{ }^{2}+3{\overline{\sigma_{\mathrm{coh}}}}^{2}\right) \sigma_{\mathrm{abs}}}{\left(\left(4-\mathrm{T}^{\prime}\right) \sigma_{\mathrm{ic}}+3 \sigma_{\mathrm{abs}}+3 \mathrm{~T}^{\prime} \sigma_{\mathrm{cos}}\right)\left(\sigma_{\mathrm{abs}}+\mathrm{T}^{\prime}\left(\sigma_{\mathrm{nc}}+\sigma_{\mathrm{col}}\right)\right)} \\
& \left(\left(4-\mathrm{T}^{\prime}\right) \sigma_{\text {inc }}+3 \sigma_{\text {abs }}+3 \mathrm{~T}^{\prime} \overline{\sigma_{\text {con }}}\right)\left(\sigma_{\text {abs }}+\mathrm{T}^{\prime}\left(\sigma_{\text {inc }}+\overline{\sigma_{\text {con }}}\right)\right)
\end{aligned}
$$

where $\sigma_{\text {abs }}$ is the neutron absorption cross-section and $\overline{\sigma_{\text {coh }}}$ is the coherent cross-section averaged over the wave vector range accessible by the instrument (see Table 2). The strength of the correction is controlled by $\mathrm{T}^{\prime}$ the transmission probability of once scattered neutrons. F or low transmissions as encountered here, it has to be considered that this value is not equal to the measured transmission of the incident neutrons $T$. $\mathrm{T}^{\prime}$ was calculated by means of an approximation explained in ref 14 from the measured T values (see Table 2).

(2) It has to be taken into account that a part of the scattering is inelastic but there is no energy discrimination at the detector. For the low neutron energies used here the relation between scattering angle $\theta$ and scattering vector $\mathrm{Q}$ is strongly energy dependent. Therefore, the integration of different energy transfers at constant angle performed in the detector is not identical to the integral at constant $\mathrm{Q}$ which is the desired result. For this, the Placzek correction has been devised. ${ }^{11,12}$ This approximate correction relies on a series expansion of the exact result in powers of $m / M$, where $m$ is the neutron and $M$ the sample atom mass. Using the correction formulas from ref 12 , we obtained unphysical results. We attribute this mainly to a failure of the 
Table 2. Parameters of the Four PI Species Relevant for the Evaluation and Correction of D7 Data ${ }^{\mathrm{a}}$

\begin{tabular}{|c|c|c|c|c|}
\hline & PIh8 & PId3 & PId5 & Pld8 \\
\hline true proton content (protons/monomer) & 7.9988 & $5.16 \pm 0.03$ & $3.09 \pm 0.02$ & $0.155 \pm 0.003$ \\
\hline coherent scattering cross section (b/monomer) & 41.81 & 50.64 & 59.38 & 69.83 \\
\hline spin-incoherent scattering cross section (b/monomer) & 642.08 & 419.96 & 258.34 & 28.52 \\
\hline isotope-incoherent scattering cross section (b/monomer) & 0.02 & 2.08 & 1.26 & 2.07 \\
\hline absorption cross section (b/monomer) & 4.49 & 2.91 & 1.76 & 0.12 \\
\hline transmission T of D7 sample & 0.836 & 0.802 & 0.802 & 0.899 \\
\hline effective film thickness calcd from transmission (mm) & 0.10 & 0.18 & 0.27 & 0.42 \\
\hline transmission $\mathrm{T}^{\prime}$ of neutrons after first scattering & 0.834 & 0.809 & 0.813 & 0.901 \\
\hline corr factor A & 1.154 & 1.197 & 1.218 & 1.176 \\
\hline corr factor B (b/srad/monomer) & 3.08 & 2.38 & 1.51 & 0.37 \\
\hline $\begin{array}{l}\text { average coherent scattering cross section } \overline{\sigma_{\text {coh }}} \\
\quad((\mathrm{b} / \mathrm{srad}) / \text { monomer })\end{array}$ & 4.35 & 4.78 & 5.60 & 4.68 \\
\hline inelasticity coeff a & $-0.052 \pm 0.003$ & $-0.032 \pm 0.005$ & $-0.047 \pm 0.003$ & $-0.020 \pm 0.003$ \\
\hline inelasticity coeff b & $0.0022 \pm 0.0002$ & $0.0011 \pm 0.0003$ & $0.0019 \pm 0.0002$ & $0.0009 \pm 0.0002$ \\
\hline
\end{tabular}

a The first five rows contain the cross sections resulting from the atomic composition. In the next three rows the data related to the transmission values measured on D7 can be found. Then follow the three parameters used forthe correction formula (eq 2) and finally the coefficients of the polynomial used for the inelasticity correction.

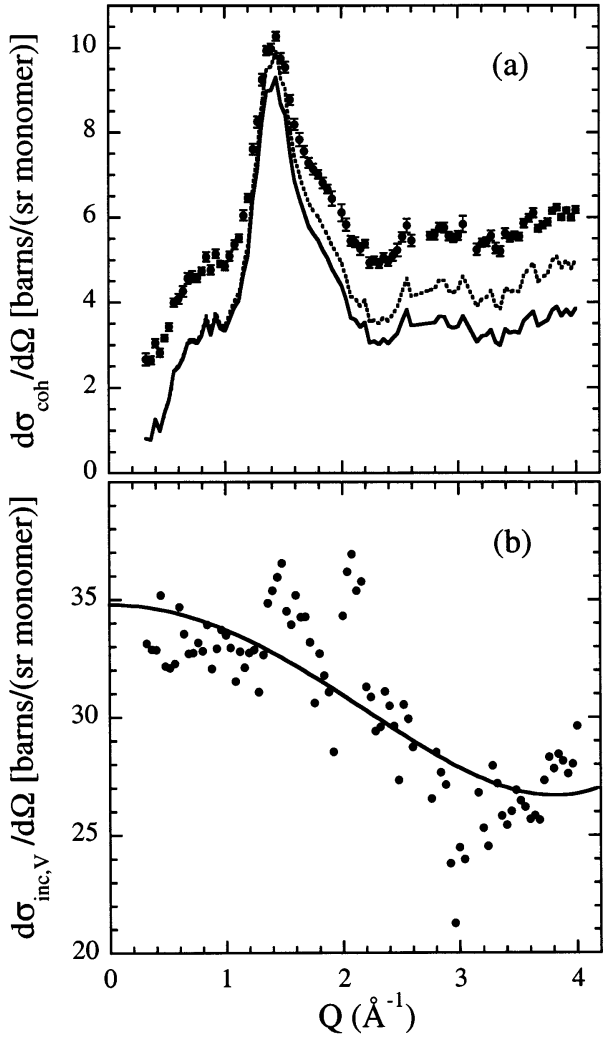

Figure 1. (a) Differential coherent neutron scattering cross sections from D7 for PId3 sample at $100 \mathrm{~K}$. The full symbols with error bars show the raw data obtained using eq 1 only with a background correction. The dotted line represents data after multiple scattering correction by eq 2 . The continuous curve shows the final data treatment stage after the inelasticity cor rection as described in the text. (b) Vanadium normalized differential incoherent neutron cross-section from D7 (PIh8, 100 K). The continuous curve is a fit with a biquadratic polynomial.

series expansion because the mass of the scattering particle (proton) $\mathrm{M}$ and $\mathrm{m}$ are about the same, and therefore, $\mathrm{m} / \mathrm{M}=1$.

For this reason we chose an empirical approach to correct the data for inelasticity effects. Figure lb shows as an example the measured differential incoherent neutron scattering cross section of PI h8 calculated from the spin-flip part of the scattered neutrons using a vanadium calibration. I deally, this should be Q-independently equal to $\sigma_{\text {ind }} / 4 \pi$. But one can clearly see that there is a $\mathrm{Q}$ dependence, which is related to the inelasticity effect on the incoherent scattering. The fit curve in Figure $1 b$ shows that it can be described reasonably by the expression $\mathrm{C}\left(1+\mathrm{aQ}^{2}+\mathrm{bQ}^{4}\right)$. Here $\mathrm{C}$ is a general intensity factor, which may also be influenced by the uncertainty of vanadium calibration, but $a$ and $b$ describe the inelasticity effect (see Table 2). Because in the calculation of the coherent differential cross-section by eq 1 the incoherent scattering shows up in the denominator, the result of (2) has to be multiplied by $\left(1+\mathrm{bQ}^{2}+\mathrm{cQ}^{4}\right)$ from the fit. Of course, the inelasticity should also be present in the numerator, the coherent scattering, but there it is less important because the involved masses (of deuterons and carbon nuclei) are larger.

(3) Finally, the derivation of eq 1 assumes that the incoherent scattering is purely due to spin disorder. Incoherent scattering due to isotopic di sorder shows up as coherent because it does not imply a spin flip of the scattered neutron. We calculated the amount of this scattering from the incompleteness of deuteration known from NMR. For all species, it is less than $0.2 \mathrm{~b} / \mathrm{srad}$, which is negligible in comparison to other error sources. Therefore, the data were not corrected for this effect.

\section{Simulation Method: Model and Simulation}

The simulations were carried out by using the I nsight (Insight II 4.0.0 P version) and the Discover-3 module from Molecular Simulations Inc. (now Accelrys) with the Polymer Consortium Force Field (PCFF). ${ }^{15}$ Most parameters of this field were derived based on ab initio data using a least-squaresd-fit technique devel oped by Hagler and co-workers. ${ }^{16}$ The functional form includes terms which can be divided into two categories-valence terms including diagonal and off-diagonal cross-coupling terms and nonbonded interactions terms. The valence terms represent internal coordinates of bond, angle, torsion angle, and out of plane angle, and the crosscoupling terms include combinations of two or three internal coordinates. The cross-coupling terms are important for predicting vibration frequencies and structural variations associated with conformational changes. The analytical expression employs quartic polynomials for bond stretching and angle bending and a threeterm Fourier expansion for torsions. The nonbonded interaction terms include a Coulombic function for the electrostatic interaction and a Lennard-J ones 6-9 function rather than the more customary LennardJ ones 6-12, for the van der Waals term. More information about this kind of force fields, including the 
complete analytical expression for the functional form, can be found in refs 15 and 16 .

The model system was built by means of the wellknown Amorphous Cell protocol, which was proposed for the first time by Theodorou and Suter.$^{17}$ In this work, a cubic cell containing one polymer chain of 100 monomer units $\left[-\mathrm{CH}_{2}-\mathrm{CH}=\mathrm{C}\left(\mathrm{CH}_{3}\right)-\mathrm{CH}_{2}-\right]_{100}$ was constructed, first of all, at $363 \mathrm{~K}$ and a density $(\rho=0.869$ $\mathrm{g} / \mathrm{cm}^{3}$ ) which was extrapolated to $363 \mathrm{~K}$ from the available experimental data. ${ }^{18}$ Such a density leads to a cell dimension of $23.53 \AA$ of side. Periodic boundary conditions were assumed in order to model the bulk system. Standard minimization procedures (PolakRibiere conjugate gradients method) were followed in order to minimize the so obtained energy structure, and subsequent dynamics was run for 1 ns in order to equilibrate the sample. The chosen temperature is high enough to allow local structural equilibration of the sample in this time. ${ }^{19}$ The system obtained in this way was used as a starting point for collecting data every 0.01 ps during a MD run of 1 ns. As integration method we have used the vel ocity-Verlet algorithm with a time step of $1 \mathrm{fs}$. The simulations were carried out in the NVT ensemble. However, instead of a real temperature-bath coupling (Nosé-Hoover or Berendsen thermostats, for instance) in order to control the temperature, we have followed a rather crude velocity scaling procedure but with a wide temperature window of $10 \mathrm{~K}$. Under these conditions, greater temperature fluctuations are allowed but the trajectory is disturbed less. In fact, we have checked that by following this simple procedure we obtain results similar to those obtained with a NVE ensemble, which has the proper Newtonian dynamics. Moreover, it is noteworthy that in a previous work ${ }^{7}$ we checked that the temperature control method used in this work also gives a vibrational density of states similar to that obtained by using the Nosé-Hoover thermostat in this kind of polyisoprene models. After the first 1 ns MD run, two more successive runs of 2 and 20 ns were carried out, collecting data every 0.05 and 0.5 ps respectively. Nearly indistinguishable results were obtained from the different simulation runs. Thus, no signature of any aging process was observed during the successive runs confirming local equilibration of the sample. In addition, a different cell was also constructed by the same protocol and equilibration procedure but starting from a different conformation of the parentchain. The results obtained for both structural and dynamic magnitudes were similar to those obtained with the first cell within the estimated uncertainties. ${ }^{8}$ A similar procedure was followed at other temperatures in the range 314-500 K. Finally, to compare the partial static structure factors calculated from our simulation cell to actual neutron scattering data (see section 4) the system was suddenly quenched to the glassy state at the same temperature (100 K) at which the D7 measurements were carried out. The density of the system was then adjusted to the estimated value at $100 \mathrm{~K}(\rho=$ $0.976 \mathrm{~g} / \mathrm{cm}^{3}$ ) by changing the cubic cell edge to $22.63 \AA$ and correspondingly scaling all atomic coordinates. After a minimization procedure similar to that described at $363 \mathrm{~K}$, a long dynamic run of 10 ns was carried out in order to accommodate the change in density.

Starting from our simulation results at the different temperatures, we have calculated the coherent differential cross-section $I_{\text {coh }}(Q)$ measured by neutron scattering with polarization analysis (D7 measure- ments) in the different samples. For an isotropic sample and taking the orientational averaging, $I_{\text {coh }}(Q)$ is defined as: ${ }^{20}$

$$
\mathrm{I}_{\text {coh }}(\mathrm{Q})=\frac{\mathrm{d} \sigma_{\text {coh }}}{\mathrm{d} \Omega}=\frac{1}{\mathrm{~N}} \sum_{\mathrm{i}, \mathrm{j}=1}^{\mathrm{N}}\left\langle\mathrm{b}_{\mathrm{i}}\right\rangle\left\langle\mathrm{b}_{\mathrm{j}}\right\rangle \frac{\sin \left(\mathrm{Qr}_{\mathrm{ij}}\right)}{\mathrm{Qr}_{\mathrm{ij}}}
$$

where the $\left\langle b_{\mathrm{i}}\right\rangle$ stand for the nuclear scattering lengths for neutrons $\left(\left\langle b_{H}\right\rangle=-0.3741 \times 10^{-14} \mathrm{~m} ;\left\langle b_{c}\right\rangle=0.6648\right.$ $\left.\times 10^{-14} \mathrm{~m} ;\left\langle\mathrm{b}_{\mathrm{D}}\right\rangle=0.6674 \times 10^{-14} \mathrm{~m}\right)$ and $\mathrm{N}$ is the number of atoms (the cross sections like $\sigma_{\text {coh }}$ are given in units of b/atom, and $I_{\text {coh }}(Q)$ is in units of b/(sr atom), $\left.1 \mathrm{~b}=10^{-28} \mathrm{~m}^{2}\right)$. In each case, $I_{\text {coh }}(\mathrm{Q})$ is calculated from the atomic coordinates of the corresponding simulation run by means of expression 3 and averaging it for a large number of frames throughout the atomic trajectories. Since the scattering lengths of carbon and deuteron atoms are very similar, these two atoms are almost indistinguishable for neutrons. In this context, for the case of the fully deuterated sample (PId8) the coherent intensity measured-or calculated-results to be just proportional to the static structure factor $S(Q)$. In the other samples, the coherent intensity given by expression 3 is just what is known as partial static structure factor.

However, it is noteworthy that what is measured in a neutron diffraction experiment without polarization analysis (D20 measurements for instance) is the total differential scattering cross-section given by

$$
\mathrm{I}(\mathrm{Q})=\frac{\mathrm{d} \sigma}{\mathrm{d} \Omega}=\frac{\mathrm{d} \sigma_{\mathrm{coh}}}{\mathrm{d} \Omega}+\frac{\mathrm{d} \sigma_{\mathrm{inc}}}{\mathrm{d} \Omega}
$$

where $\mathrm{d} \sigma_{\text {ind }} d \mathrm{~d} \Omega$ is a Q-independent incoherent term defined as

$$
\mathrm{I}_{\mathrm{inc}}=\frac{\mathrm{d} \sigma_{\mathrm{inc}}}{\mathrm{d} \Omega}=\frac{1}{\mathrm{~N}} \sum_{\mathrm{i}=1}^{\mathrm{N}}\left(\left\langle\mathrm{b}_{\mathrm{i}}{ }^{2}\right\rangle-\left\langle\mathrm{b}_{\mathrm{i}}\right\rangle^{2}\right)=\frac{\sigma_{\mathrm{inc}}}{4 \pi}
$$

For a fully deuterated sample of $\mathrm{PI}$, the value of $\mathrm{I}_{\text {inc }}$ is $0.10 \mathrm{~b} /(\mathrm{sr}$ atom). Therefore, to compare the simulation results with the diffraction measurements on PId8 carried out by means of the D20 instrument at different temperatures, we have al so cal culated I (Q) by means of expressions 3-5 and, again, averaging it for a large number of frames.

\section{Results}

First of all, we will describe the results obtained by means of the D20 diffractometer on the fully deuterated sample PId8. As it has already been mentioned in the Experimental Section, D20 does not provide polarization analysis; i.e., it measures the total scattering I (Q) given by eq 4 . The I (Q) results on PId8 are dominated by the coherent contribution, which as it has been abovementioned, is just proportional to the static structure factor (see section 3). The main advantage of the D20 instrument is its ability to cover a wide $\mathrm{Q}$ range extending up to $Q \approx 13 \AA^{-1}$. This allows scrutinizing how the structure of the simulated cell reproduces the short intrachain correlation details. On the other hand, as D20 is a high-flux instrument, a wide temperature range can easily be covered with reasonable measuring times.

After the corrections described in section 2, the results obtained at $100 \mathrm{~K}$ are shown in Figure 2 in comparison 


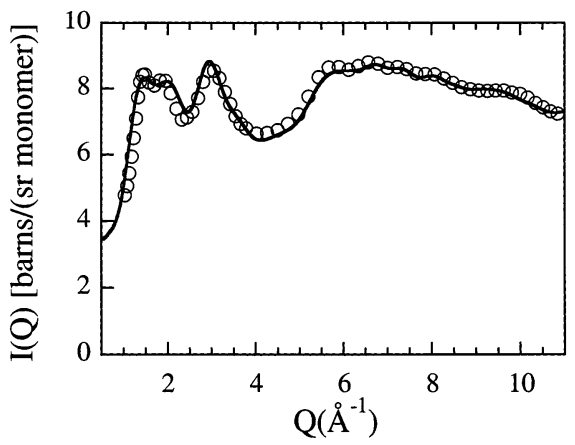

Figure 2. Total differential scatteringcross section I(Q) obtained from D20 measurements at $100 \mathrm{~K}$ for a fully deuterated sample (PId8) (O) and calculated from the simulations at the same temperature (solid line).

with the I (Q) calculated at the same temperature from the atomic trajectories by means of eqs $3-5$. The scale of I(Q) in Figure 2 is the absolute scal e corresponding to the data from simulations and which gives the correct high Q-limit. As it was already mentioned in the Experimental Section, after the "calibration" made by comparison with D7 data, the corrected D20 intensities are given in absolute units. The experimental I(Q) shows two first overlapping peaks of about the same intensity in the so-called "intermolecular $\mathrm{Q}$ range". These peaks are centered at $\mathrm{Q} \sim 1.4 \AA^{-1}$ and $\mathrm{Q} \sim 1.9 \AA^{-1}$ respectively. A third peak, also of about the same intensity, appears at a higher value of $Q$ of about $3 \AA^{-1}$. This peak seems to be a universal feature of all amorphous polymers and, since it does not shift with temperature, it has been associated with intrachain correlations (covalent bonds). At $\mathrm{Q}$ values higher than $4 \AA^{-1}, \mathrm{I}(\mathrm{Q})$ displays different peaks which should be attributed to short distance intrachain atomic correlations. As can be seen in Figure 2, in the full Q range the agreement between experimental and simulation data is good. Nonequilibrium effects of the quenched cell at $100 \mathrm{~K}$ and the experimental uncertainties from multiple scattering and inelasticity effects obviously are well controlled. From these first results, we can conclude that the average structural information which is contained in the static structure factor or I(Q) is well reproduced by the simulation cell at least at $100 \mathrm{~K}$.

The temperature evolution of I (Q) only shows significant changes at $Q$ values lower than $Q \sim 3 \AA^{-1}$ ("intermolecular" Q range). Therefore, in the following we will mainly concentrate in this range. Figure 3 displays experimental I(Q) data corresponding to different temperatures in the range $2-314 \mathrm{~K}$. Simulation data in the available high-temperature range 314-500 $\mathrm{K}$ are also included in the figure for comparison. The two first peaks of I (Q) shift with temperature to lower $Q$ values confirming the interchain origin of the peaks. In contrast, the peak centered at $Q \sim 3 \AA^{-1}$ does not evolve with temperature-as in other amorphous polymers-indicating a pure intramolecular origin of this peak.

In Figure 4, we represent the temperature behavior of the mean "interchain" distance, $d$, corresponding to the first peak of I(Q). This distance is defined as $d=$ $2 \pi / Q_{\max }$, where $Q_{\max }$ is the main peak position. The figure includes data from both experiments and simulations. There are only two temperatures (100 and 314 $\mathrm{K})$ at which $\mathrm{d}$ can be calculated from both sets of data. However, the plot shows that there is a very good agreement between the temperature dependence of $d$

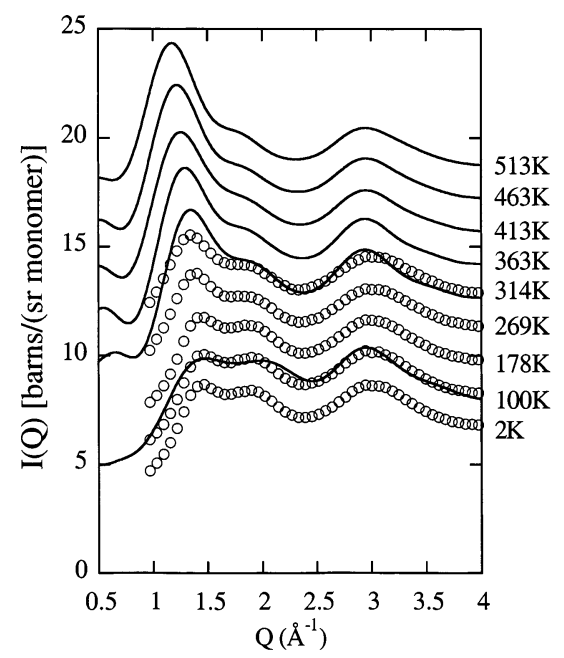

Figure 3. Temperature evolution of the total differential scattering cross section I(Q) obtained from D20 measurements for a fully deuterated sample (PId8) (O) and calculated from the simulations (solid lines). The scale of I (Q) indicated in the figure corresponds to the experimental curve at $2 \mathrm{~K}$. The curves corresponding to other temperatures have been shifted vertically 1.5 units each for clarity.

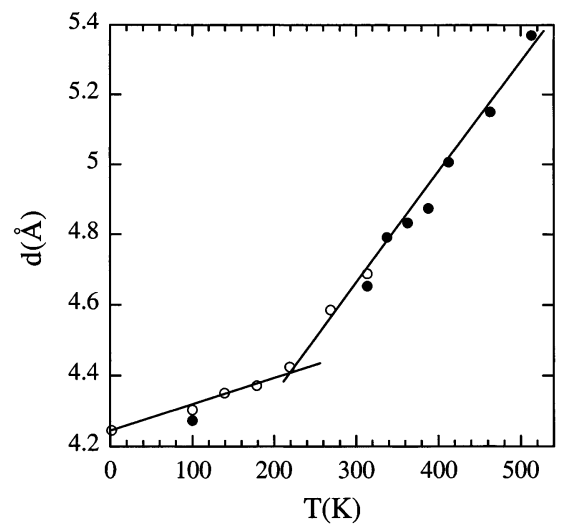

Figure 4. Temperature dependence of the main "interchain" distance corresponding to the first peak of I (Q) shown in Figure 3 . This distance is defined as $d=2 \pi / Q_{\max }$, where $Q_{\max }$ is the main peak position. E mpty points correspond to experimental data and full points to simulations.

calculated either from experimental data or from simulations. $d(T)$ shows two different linear regimes crossing over at the temperature range of the calorimetric glasstransition of $\mathrm{PI}\left(\mathrm{T}_{\mathrm{g}} \sim 200 \mathrm{~K}\right)$. This is a typical behavior already observed for different amorphous polymers. ${ }^{2}$ Figure 3 also shows that the intensity of the first peak of the experimental I (Q) is significantly increasing with temperature and this effect al ready starts at temperatures well below the glass transition of PI. This experimental trend is also nicely captured by the simulation results (see Figure 3). However, there is a slight discrepancy between the absolute value of the maximum intensity of experimental and simulated I(Q) at $314 \mathrm{~K}$, which is the only temperature at which both sets of data can be compared concerning this effect.

Now we will concentrate on the results obtained by means of D7 spectrometer with polarization analysis, providing the coherent contribution to the scattering on an absolute scale. Therefore, not only fully deuterated samples-where the scattering is dominated by the coherent contribution-but also partially deuterated samples and even fully protonated samples, can be measured. However, the $Q$ range is limited by $4 \AA^{-1}$. 

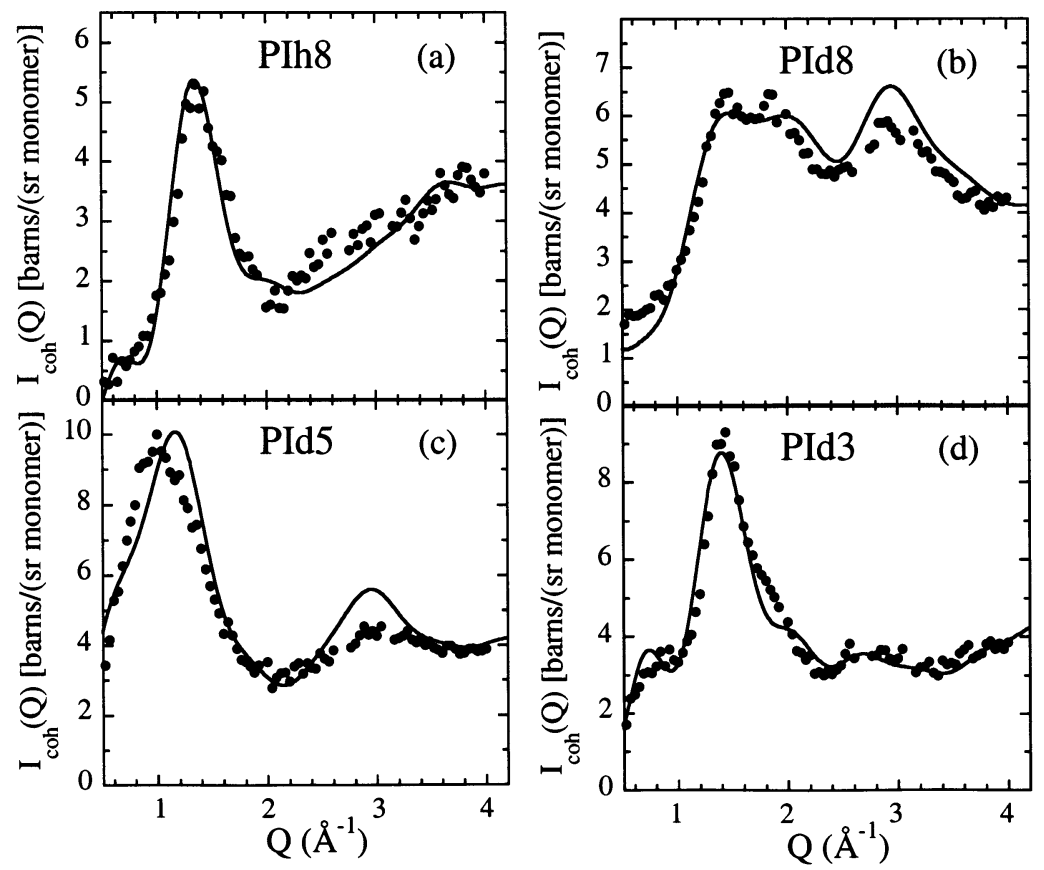

Figure 5. Differential coherent neutron scattering cross sections $I_{\text {coh }}(Q)$ obtained from $D 7$ measurements at $100 \mathrm{~K}$ for the four samples investigated in comparison with the simulation results. Full points are experimental data. Continuous lines correspond to the simulations.

Figure 5 presents the corrected results obtained at 100 $\mathrm{K}$ for the four samples investigated. F or $\mathrm{PI} d 8, \mathrm{I}_{\text {coh }}(\mathrm{Q})$ is very similar to the total scattering I(Q) measured by D20 because in this case I(Q) is dominated by the coherent contribution. However, in the case of the other samples, the situation is different and $I_{\text {coh }}(Q)$ only displays one main peak in the interchain $\mathrm{Q}$ region which is centered at different $Q$ values: $Q \sim 1.3 \AA^{-1}$ (PIh8); $\mathrm{Q} \sim 1.5 \AA^{-1}$ (PI d3); $\mathrm{Q} \sim 1 \AA^{-1}(\mathrm{PId} 5)$. In addition, $\mathrm{I}_{\text {coh }}(\mathrm{Q})$ corresponding to the PId3 sample exhibits a clear shoulder in the low $\mathrm{Q}$ range $\mathrm{Q}<1 \AA^{-1}$. The shape of the main peak of the PId5 sample also indicates that some low-Q shoulder could be hidden bel ow this main peak. Moreover, as in the case of $\mathrm{PId} 8$ sample, $\mathrm{I}_{\text {coh }}(\mathrm{Q})$ of PId5 also shows a small peak at $Q \sim 3 \AA^{-1}$. Figure 5 also includes the simulated results of $I_{\text {coh }}(Q)$ for the four samples at $100 \mathrm{~K}$. The agreement between experimental and simulated data is almost perfect in the case of the PIh8, PId3, and PId8 samples (in the last case the comparison was already done in terms of I(Q)). In the case of the PId5 sample, the main peak from the simulations is slightly shifted to higher $\mathrm{Q}$ values. Also, the intensity of the peak centered at $3 \AA^{-1}$ is overestimated by the simulation data. In any case, we can say that the agreement found between simulation and experimental results is remarkable, taking into account that we are extending the comparison beyond the simple static structure factor.

\section{Discussion}

a. Partial Structure Factors. From the results described in the previous section we can conclude that the inter-and intramacromol ecular structure obtained in our simulation cell constitutes a quite reasonable representation of the actual structure of $\mathrm{PI}$. Now we can take advantage of the information contained in the simulation data and try to understand the main atomic correlations contributing to the different peaks of the different partial structure factors measured and calculated. To do this, we have to consider separately the different atomic correlations in the sample cell. There fore, we have calculated the set of partial radial distribution functions $\mathrm{G}_{\mathrm{CC}}(r), \mathrm{G}_{\mathrm{HH}}(r)$, and $\mathrm{G}_{\mathrm{CH}}(r)$ where $\mathrm{C}$ and $\mathrm{H}$ denote carbon and hydrogen (deuteron), respectively. Moreover, in each case we have considered separately main-chain carbons or hydrogens (deuterons) and methyl group carbon or hydrogens (deuterons). This implies that we are working with 10 different partial radial distribution functions. On the other hand, as our main interest is to unravel the different atomic correlations contributing to the different peaks of the partial static structure factors, we will discuss our results in the reciprocal Q-space instead of the most customary real space. Therefore, we will consider the Fourier transform of $\mathrm{G}_{\alpha \beta}(\mathrm{r})[\alpha, \beta$ : $\mathrm{C}, \mathrm{H}]$, i.e., the different contributions to the eqs 3 corresponding to the different atomic correlations in real space. First of all, we will discuss these contributions-which were properly normalized in order to take into account the number of the different atomic species-neglecting for the moment the different neutron scattering lengths. We will name them $A_{\alpha \beta}(Q)$. In the next section, we will see how these contributions are highlighted or downgraded in the different samples according to the different neutron scattering lengths.

Figure 6a shows the different carbon-carbon correlations $A_{c c}(Q)$ in the rel evant $Q$ range up to $4 \AA^{-1}$. There are three different contributions: $A_{\text {Cchcch }}$ (dashed line), which corresponds to carbon chain-carbon chain correlations; $A_{c c h c m}$ (dashed dotted line), which corresponds to carbon chain-carbon methyl correlations; $A_{C m c m}$ (dotted line), which corresponds to carbon methyl-carbon methyl correlations. The most relevant feature is that in the $\mathrm{Q}$ range considered the total carbon-carbon correlation function $A_{C c}(Q)$ (solid line) is dominated by the $A_{C c h c c h}$ contribution. Moreover, $A_{C C}(Q)$ only shows one clear and intense peak centered at about $\mathrm{Q} \sim 1.3$ $\AA^{-1}$, i.e., just in the $Q$ range where the coherent intensity, I coh (Q), of PIh8 sample shows its first peak. We will come back to this question below. It is note- 


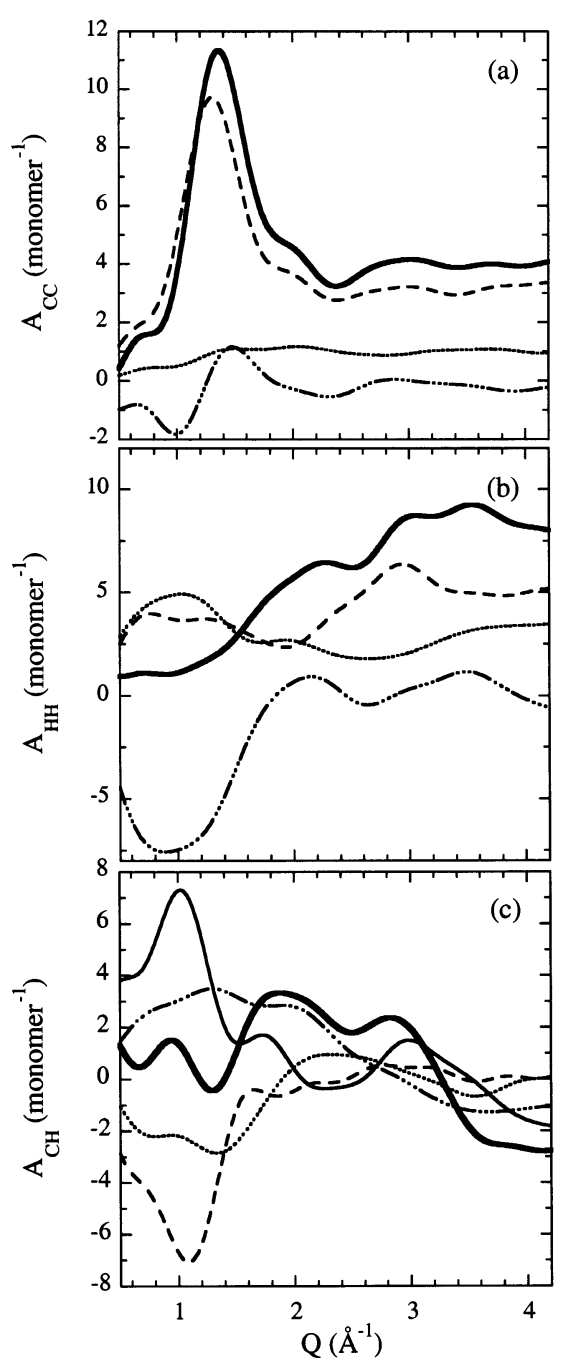

Figure 6. Fourier transform of different partial radial distribution functions corresponding to different atomic correlations (see the text for definitions). (a) Key: carbon-carbon correlations: total $A_{c c}$ (continuous line); carbon chain-carbon chain $A_{c c h c c h}$ (dashed line); carbon methyl-carbon methyl $A_{c m c m}$ (dotted line); carbon chain-carbon methyl $A_{c c h c m}$ (dasheddotted line). (b) Key: hydrogen-hydrogen correlations: total $\mathrm{A}_{\mathrm{HH}}$ (continuous line); hydrogen chain-hydrogen chain $\mathrm{A}_{\mathrm{HchHc}}$ (dashed line); hydrogen methyl-hydrogen methyl $A_{H \mathrm{HHm}}$ (dotted line); hydrogen chain-hydrogen methyl $A_{H c h H m}$ (dasheddotted line). (c) Key: carbon-hydrogen correlations: total $A_{C H}$ (continuous thick line); carbon chain-hydrogen chain $A_{c c h H c h}$ (continuous thin line); carbon chain-hydrogen methyl AcchHm (dashed line); carbon methyl-hydrogen chain $A_{c m H c h}$ (dotted line); carbon methyl-hydrogen methyl $A_{\mathrm{CmHm}}$ (dashed-dotted line).

worthy that the contribution to $I_{\text {coh }}(Q)$ coming from $A_{c c}(Q)$ is the same for all the samples investigated and just given by $\left\langle b_{c}\right\rangle^{2} A_{c c}(Q)$.

The different hydrogen-hydrogen correlations $\mathrm{A}_{\mathrm{HH}}(\mathrm{Q})$ are shown in Figure $6 \mathrm{~b}$. The hydrogen chain-hydrogen methyl contribution, $A_{\mathrm{HchHm}}$ (dashed dotted line) shows a strong negative peak in the $Q$ region around $1 \AA^{-1}$ but becomes al most zero at $Q \geq 2 \AA^{-1}$. Another salient feature is that the hydrogen chain-hydrogen chain correlations $A_{H c h H c h}$ (dashed line) feature a relatively strong peak centered at $Q \sim 3 \AA^{-1}$ where the $I_{\text {coh }}(Q)$ of many amorphous polymers show a peak which does not evolve with temperature. As can be seen in the figure, the total hydrogen-hydrogen correlations $A_{H H}(Q)$ (solid line) do not display significant values in the $\mathrm{Q}$ region $\mathrm{Q}$ $<1.5 \AA^{-1}$, where the carbon-carbon correlations exhibit their main contribution (see Figure 6a). It is important to point out that the contribution to $\mathrm{I}_{\mathrm{coh}}(\mathrm{Q})$ coming from $\mathrm{A}_{\mathrm{HH}}(\mathrm{Q})$ will be the same for PI h8 and PId8 (apart from a factor: $\left\langle b_{H}\right\rangle^{2}$ in the case of PIh8 and $\left\langle b_{D}\right\rangle^{2}$ for PId8). However, this contribution will be different in the case of PId5 and PId3 samples.

Figure $6 c$ shows the different carbon-hydrogen correlations $A_{C H}(Q)$. As can be seen, the different contributions show a complicated $\mathrm{Q}$ dependence. Both, carbon chain-hydrogen chain $\left(A_{c c h H c h}\right)$ (continuous thin line) and carbon chain-hydrogen methyl $\left(\mathrm{A}_{\mathrm{c}} \mathrm{hH} \mathrm{m}\right)$ (dashed line) correlations, show a strong peak centered on $\mathrm{Q} \sim$ $1 \AA^{-1}$. However, since these two peaks have opposite signs, they compensate each other giving an almost null contribution to the total $A_{C H}(Q)$. The same happens with the carbon methyl-hydrogen chain $\left(\mathrm{A}_{\mathrm{CmHch}}\right)$ (dotted line) and carbon methyl-hydrogen methyl $\left(\mathrm{A}_{\mathrm{cmHm}}\right)$ (dashed dotted line) correlations. Therefore, we do not expect a significant contribution to $\mathrm{I}_{\text {coh }}(\mathrm{Q})$ from carbonhydrogen cross-correlations at $\mathrm{Q}<1.5 \AA^{-1}$ either in the fully hydrogenated (PIh8) or in the fully deuterated (PId8) samples. The situation is different in the case of the partially deuterated PId5 and PId3 samples.

b. Experimental Results in Terms of Partial Structure Factors. In Figure 5, we have demonstrated the good agreement between the experimentally determined structure factors from the differently labeled samples and the simulations results. Now we like to take the simulations, to understand how the different features in the experimental results related to the partial structure factors. For that purpose we display how the corresponding simulated structure factors are built up by the specific atom-atom correlations $A_{\alpha \beta}(Q)$. This knowledge will be later important to rationalize also the temperature dependencies.

To discuss how the different $A_{\alpha \beta}(Q)$ contribute to the coherent scattering $I_{\text {coh }}(Q)$ from the different samples we have to weight them properly by the corresponding neutron scattering lengths $\left\langle b_{H}\right\rangle_{,}\left\langle b_{c}\right\rangle$, and $\left\langle b_{D}\right\rangle$ respectively.

(1) Fully Protonated Sample PIh8. Figure 7a presents the contributions coming from the $A_{\alpha \beta}(Q)$ terms for the fully protonated sample PIh8. It is evident that the main peak in this case results from the intensity coming from carbon-carbon correlations: $I_{c c}(Q)=\left\langle b_{c}\right\rangle^{2} A_{c c}(Q)$ (dotted line). Moreover, taking into account the previous discussion on $A_{c c}(Q)$, we conclude that the main contribution to this peak originates from the carbon chain-carbon chain correlations. On the other hand, Figure 7a also shows that the continuous increase of intensity displayed by $\mathrm{I}_{\mathrm{coh}^{-}}$ (Q) for $\mathrm{Q}>2.5 \AA^{-1}$ can be assigned to a combination of carbon-carbon, Icc(Q) (dotted line), hydrogenhydrogen $\left[\mathrm{I}_{\mathrm{HH}}(\mathrm{Q})=\left\langle\mathrm{b}_{\mathrm{H}}\right\rangle^{2} \mathrm{~A}_{\mathrm{HH}}(\mathrm{Q})\right]$ (dashed line) and carbon-hydrogen $\left[\mathrm{I}_{\mathrm{CH}}(\mathrm{Q})=\left\langle\mathrm{b}_{\mathrm{C}}\right\rangle\left\langle\mathrm{b}_{\mathrm{H}}\right\rangle \mathrm{A}_{\mathrm{CH}}(\mathrm{Q})\right]$ (dashed dotted line) contributions.

(2) Fully Deuterated Sample PId8. Figure 7b shows similar data but now for the fully deuterated sample PId8. As it was al ready commented I coh (Q) from this sample is characterized by two peaks in the "intermolecular" Q range. Looking at the figure, it is now evident that the peak centered at the lower $\mathrm{Q}$ value is again almost exclusively due to carbon-carbon correlations. However, the second peak in this $\mathrm{Q}$ range cannot easily be attributed to any particular atomic correlation, being a combination of carbon-carbon, Icc $(\mathrm{Q})$, hydrogen-hydrogen [now deuteron-deuteron: 


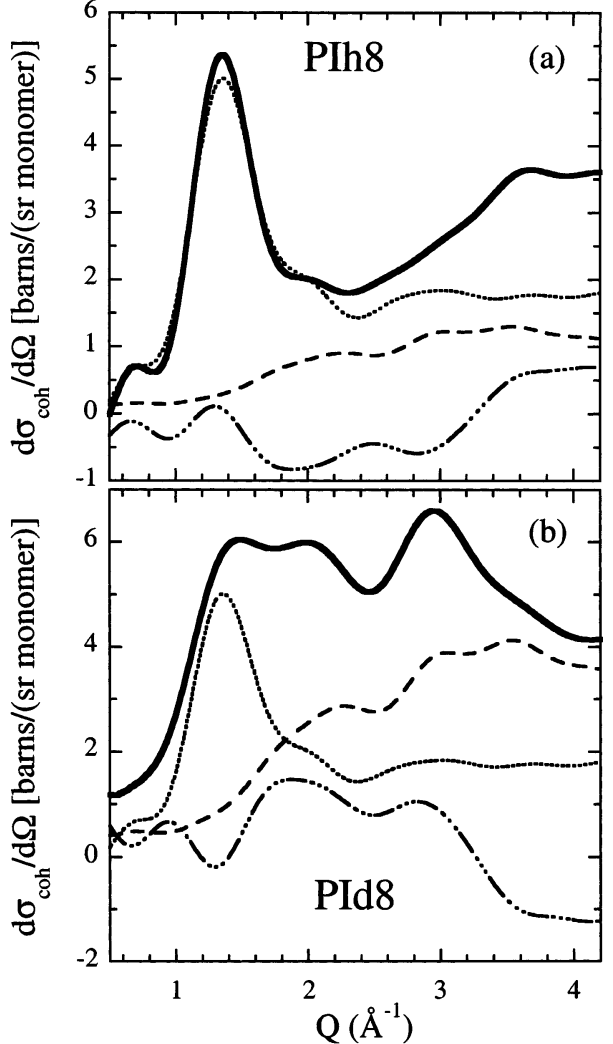

Figure 7. Contributions to the coherent scattering coming from the different atomic correlations and properly weighted by the corresponding neutron scattering lengths. (a) PIh8 sample: total coherent intensity $I_{\text {coh }}(Q)$ (continuous line); carbon-carbon correlations term I $\mathrm{cc}_{\mathrm{C}}(\mathrm{Q})$ (dotted line); hydrogenhydrogen correlations term $\mathrm{I}_{\mathrm{HH}}(\mathrm{Q})$ (dashed line); carbonhydrogen correlations term $\mathrm{I}_{\mathrm{CH}}(\mathrm{Q})$ (dashed-dotted line). (b) PI d8 sampl e: total coherent intensity $\mathrm{I}_{\text {coh }}(\mathrm{Q})$ (continuous line); carbon-carbon correlations term Icc $(Q)$ (dotted line); deuterondeuteron correlations term I $\mathrm{DD}_{\mathrm{DD}}(\mathrm{Q})$ (dashed line); carbondeuteron correlations term I CD $(Q)$ (dashed-dotted line).

$\mathrm{I}_{\mathrm{DD}}(\mathrm{Q})=\left\langle\mathrm{b}_{\mathrm{D}}\right\rangle^{2} \mathrm{~A}_{\mathrm{HH}}(\mathrm{Q})$ ] and carbon-hydrogen [now carbon-deuteron: $\left.I_{C D}(Q)=\left\langle b_{C}\right\rangle\left\langle b_{D}\right\rangle A_{C H}(Q)\right]$ contributions. From the data shown in Figure $7 \mathrm{~b}$, we can estimate the contribution of each of these correlations to that peak: carbon-carbon correlations ( $30 \%)$; deuteron-deuteron correlations ( $\sim 50 \%)$; carbon-deuteron correlations ( 20\%).

Furthermore, also the peak at about $3 \AA^{-1}$ seems to arise from a combination of contributions from the different atomic correlations. Figure $7 \mathrm{~b}$ shows that the main contribution relates to deuteron-deuteron correlations. The atomic correlations giving raise to this peak correspond to short distances and have to be intrachain correlations. We point out that this peak does not shift with temperature neither in the experimental data nor in the simulations results.

(3) Main Chain Deuterated Sample PId5. The main feature displayed by $\mathrm{I}_{\text {coh }}(\mathrm{Q})$ from PId5 sample (Figure 8a) is a peak centered at lower $Q\left(Q \sim 1 \AA^{-1}\right)$ compared to the rest of the samples studied. Moreover, this peak broadens at the low Q-side, even exhibiting a shoulder-like structure. This extra intensity in the low $\mathrm{Q}$ range, $\mathrm{Q}<1 \AA^{-1}$, cannot be due to carbon-carbon correlations because the contribution from these correlations is the same for all the samples and only shows a quite narrow peak centered at a higher $\mathrm{Q}$ value (see dotted line in Figure 8a). In a subtle way, the strong low $Q$ peak is rather made up by $(H, D)$ and $C-(H, D)$

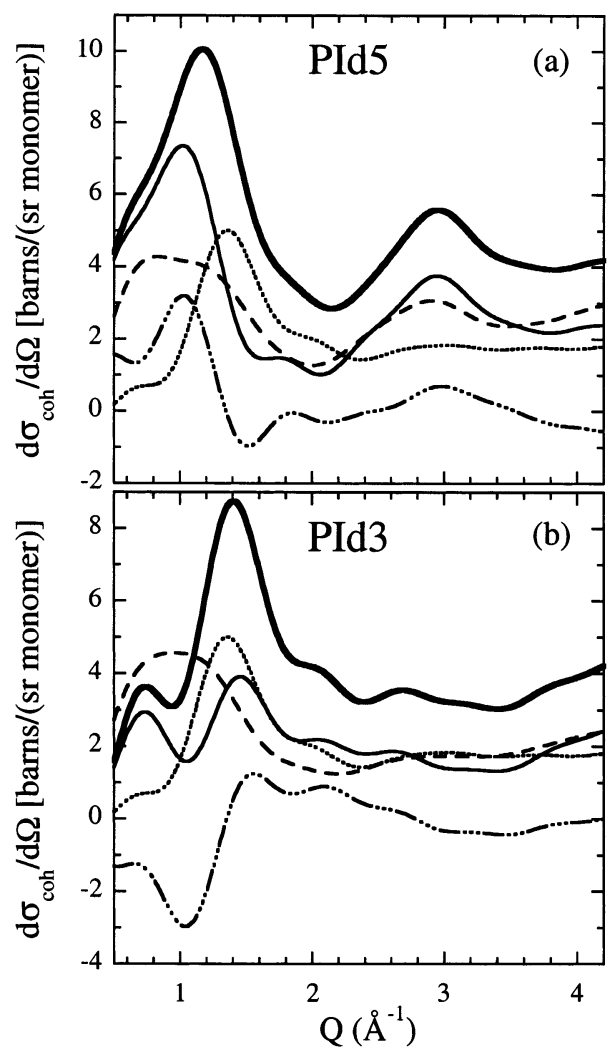

Figure 8. Contributions to the coherent scattering coming from the different atomic correlations and properly weighted by the corresponding neutron scattering lengths. (a) PId5 sample: total coherent intensity $\mathrm{I}_{\text {con }}(\mathrm{Q})$ (continuous thick line); carbon-carbon correlations term I $\mathrm{cc}(\mathrm{Q})$ (dotted line); hydrogen [or deuteron]-hydrogen [or deuteron] correlations term I $\mathrm{HD}_{\mathrm{D}}(\mathrm{Q})$ (dashed line); carbon-hydrogen [or deuteron] correlations term $I_{C H D}(Q)$ (dashed-dotted line); $I_{H D}(Q)+I_{C H D}(Q)$ (continuous thin line). (b) PId3 sample: total coherent intensity $\mathrm{I}_{\text {coh }}(\mathrm{Q})$ (continuous thick line); carbon-carbon correlations term I cc(Q) (dotted line); hydrogen [or deuteron] -hydrogen [or deuteron] correlations term I $\mathrm{HD}_{\mathrm{H}}(\mathrm{Q})$ (dashed line); carbonhydrogen [or deuteron] correlations term $\mathrm{I}_{\mathrm{CHD}}(\mathrm{Q})$ (dasheddotted line); $I_{H D}(Q)+I_{C H D}(Q)$ (continuous thin line).

correlations. Thereby the different signs of $\left\langle b_{D}\right\rangle$ and $\left\langle b_{H}\right\rangle$ play an important role. Let us first consider the intensity arising from the $(H, D)$ correlations:

$$
\begin{array}{r}
\mathrm{I}_{\mathrm{HD}}(\mathrm{Q})=\left\langle\mathrm{b}_{\mathrm{D}}\right\rangle^{2} \mathrm{~A}_{\mathrm{HchHch}}(\mathrm{Q})+\left\langle\mathrm{b}_{\mathrm{D}}\right\rangle\left\langle\mathrm{b}_{\mathrm{H}}\right\rangle \mathrm{A}_{\mathrm{HchHm}}(\mathrm{Q})+ \\
\left\langle\mathrm{b}_{\mathrm{H}}\right\rangle^{2} \mathrm{~A}_{\mathrm{HmHm}}(\mathrm{Q})
\end{array}
$$

(dashed line in Figure 8a). As can be seen from Figure 6 , the negative term $A_{\mathrm{HchHm}}$ featuring the correlations between the chain and the methyl group hydrogens transforms now into a positive contribution because the factor $\left\langle b_{D}\right\rangle\left\langle b_{H}\right\rangle$ is also negative. This positive contribution, together with that from $\mathrm{A}_{\mathrm{HchH}} \mathrm{H}$ (now highlighted by the factor $\left\langle b_{D}\right\rangle^{2}=0.44$ ) are the main responsibles for the low-Q broad positive peak of $I_{H D}(Q)$. The term $A_{H m H m}$ does not contribute very much due to the small factor $\left\langle\mathrm{b}_{\mathrm{H}}\right\rangle^{2}=0.14$.

Second, we inspect the contributions arising from the $\mathrm{C}-(\mathrm{H}, \mathrm{D})$ correlations

$$
\begin{aligned}
\mathrm{I}_{\mathrm{CHD}}(\mathrm{Q})= & \left\langle\mathrm{b}_{\mathrm{C}}\right\rangle\left\langle\mathrm{b}_{\mathrm{D}}\right\rangle \mathrm{A}_{\mathrm{CchHch}}(\mathrm{Q})+\left\langle\mathrm{b}_{\mathrm{C}}\right\rangle\left\langle\mathrm{b}_{\mathrm{H}}\right\rangle \mathrm{A}_{\mathrm{CchHm}}(\mathrm{Q})+ \\
& \left\langle\mathrm{b}_{\mathrm{C}}\right\rangle\left\langle\mathrm{b}_{\mathrm{D}}\right\rangle \mathrm{A}_{\mathrm{CmHCh}}(\mathrm{Q})+\left\langle\mathrm{b}_{\mathrm{C}}\right\rangle\left\langle\mathrm{b}_{\mathrm{H}}\right\rangle \mathrm{A}_{\mathrm{CmHm}}(\mathrm{Q})
\end{aligned}
$$

(dashed-dotted line in Figure 8a). It shows a narrow positive peak centered at $\mathrm{Q} \sim 1 \AA^{-1}$ resulting from the 
positive term $A_{C c h H c h}$ and the negative $A_{c c h H m}$ (see Figure 6) which now becomes positive because the factor $\left\langle b_{c}\right\rangle\left\langle b_{H}\right\rangle$ is negative. The other contributions are considerably smaller in the low $\mathrm{Q}$ range. Finally, the continuous thin line in Figure 8a displays the sum of $I_{H D}(Q)$ and $I_{C H D}(Q)$ featuring a strong peak in the low Q-regime. Thus, we may conclude that the peak at Q $1 \AA^{-1}$ seen in the PId5 sample is not due to carboncarbon correlations but is brought about by correlations involving $\mathrm{H}$ and $\mathrm{D}$ where the different signs of $\left\langle\mathrm{b}_{\mathrm{H}}\right\rangle$ and $\left\langle b_{D}\right\rangle$ play an essential role. On the other hand, as in the case of the PId8 sample, the peak at about $3 \AA^{-1}$ is a combination of contributions from all atomic correlations, which in this $Q$ range are of an intrachain nature.

(4) Main Chain Protonated Sample PId3. A part from the main peak at $Q \sim 1.5 \AA^{-1}$, the most relevant feature of the $\mathrm{I}_{\mathrm{coh}}(\mathrm{Q})$ is the small peak visible in the low $\mathrm{Q}$ region $\left(\mathrm{Q} \sim 0.7 \AA^{-1}\right)$ (F igure $\left.8 \mathrm{~b}\right)$. It is noteworthy that, although less defined, this peak is clearly present also in the experimental $\mathrm{I}_{\text {coh }}(\mathrm{Q})$ (Figure 5). For the PId3 sample, the carbon-carbon correlations Icc(Q) (dotted line) significantly contribute to the main peak at $\mathrm{Q} \sim$ $1.5 \AA^{-1}$. However, it is also evident that these correlations are not causing the small peak at $Q \sim 0.7 \AA^{-1}$. On the other hand, as in the case of PId5, $\mathrm{IHD}_{\mathrm{HD}}(\mathrm{Q})$ (eq 6) al so here shows a broad maximum in the low $Q$ range (dashed line). In this regime, the dominant contribution again comes from the cross term $\left\langle b_{H}\right\rangle\left\langle b_{D}\right\rangle A_{H c h H m}(Q)$. The different weighting of the other two terms $-\left\langle b_{H}\right\rangle$ and $\left\langle b_{D}\right\rangle$ are exchanged-has consequences at high $\mathrm{Q}$. There, the strong peak shown by $A_{H c h H c h}$ (see Figure 6) is now downgraded in comparison to the PId5 sample.

Compared to the PId5 sample, the $\mathrm{C}-(\mathrm{H}, \mathrm{D})$ correlations, $I_{C H D}(Q)$ appears reversed in sign. While in the case of $\mathrm{PI}$ d5 I $\mathrm{CHD}_{\mathrm{CH}}(\mathrm{Q})$ shows a positive peak at $\mathrm{Q} \sim 1 \AA^{-1}$, the peak becomes negative for PId3. The opposite happens with the peak at $\mathrm{Q} \sim 1.5 \AA^{-1}$ (see Figure 8, parts $a$ and $b$ ). This can easily be explained by comparing the expression of $\mathrm{I}_{\mathrm{CHD}}(\mathrm{Q})$ for the PId3 sample:

$$
\begin{aligned}
\mathrm{I}_{\mathrm{CHD}}(\mathrm{Q})= & \left\langle\mathrm{b}_{\mathrm{C}}\right\rangle\left\langle\mathrm{b}_{\mathrm{H}}\right\rangle \mathrm{A}_{\mathrm{CchHch}}(\mathrm{Q})+\left\langle\mathrm{b}_{\mathrm{C}}\right\rangle\left\langle\mathrm{b}_{\mathrm{D}}\right\rangle \mathrm{A}_{\mathrm{CchHm}}(\mathrm{Q})+ \\
& \left\langle\mathrm{b}_{\mathrm{C}}\right\rangle\left\langle\mathrm{b}_{\mathrm{H}}\right\rangle \mathrm{A}_{\mathrm{CmHCh}}(\mathrm{Q})+\left\langle\mathrm{b}_{\mathrm{C}}\right\rangle\left\langle\mathrm{b}_{\mathrm{D}}\right\rangle \mathrm{A}_{\mathrm{CmHm}}(\mathrm{Q})
\end{aligned}
$$

with that corresponding to PId5 sample (eq 7) and taking into account that $A_{C c h H c h}$ and $A_{C c h H m}$ are al most symmetric and $A_{\mathrm{cmHm}}$ and $A_{\mathrm{CmHch}}$ as well (see Figure 6). We conclude that, as in the case of the PId5 sample, al so for PId3 the low-Q behavior is a consequence of a balance between the $I_{H D}(Q)$ and $I_{C H D}(Q)$ contributions. This is evident from Figure $8 \mathrm{~b}$, where we also have represented the sum of these two terms (thin solid line).

It is noteworthy that the presence of small peaks in the low $\mathrm{Q}$ range bel ow the main "intermolecular" peaks in an amorphous polymer is usually taken as the signature of some kind of intermediate range order in the system. However, the previous discussion shows that at least in the case of PI this is not the case. Finally, Figure $8 \mathrm{~b}$ shows that, although all the atomic correlations contribute to the main peak of $I_{\text {coh }}(Q)$ of $P I d 3$, the most rel evant are the carbon-carbon correlations and among them the carbon chain-carbon chain correlations.

c. Temperature Dependence. Until now, we have discussed the origin of the main peaks featured by the different partial static structure factors $I_{\text {coh }}(Q)$ at 100 $\mathrm{K}$. Now, we come back to the question of the temperature evolution of the static structure factor (PId8

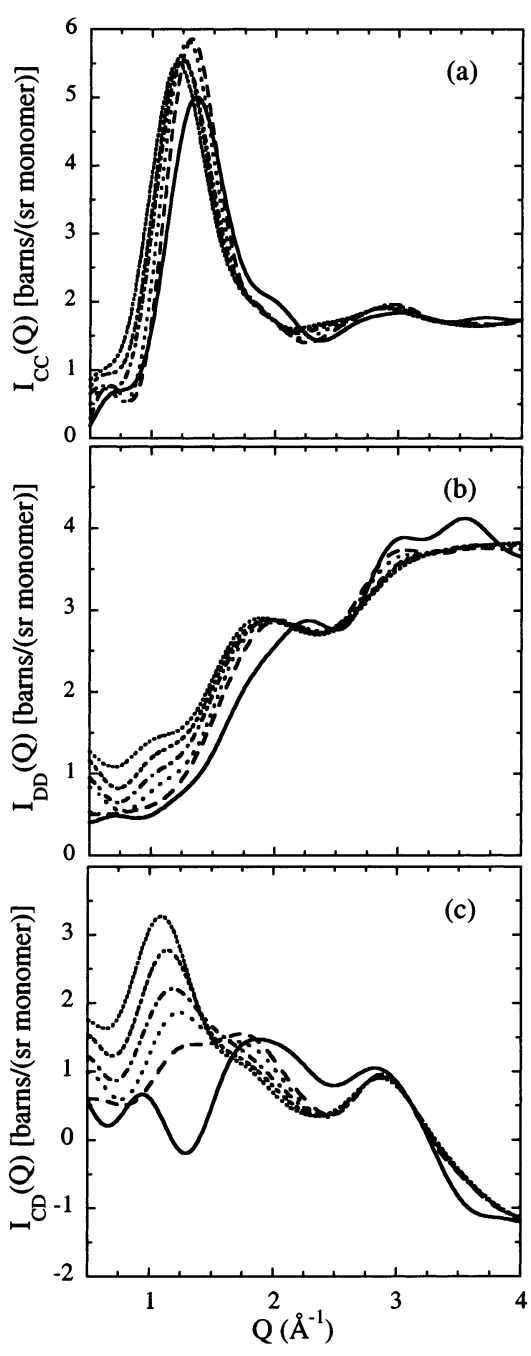

Figure 9. Temperature evolution of the contributions to the coherent scattering coming from the different atomic correlations in the case of PId8 sample. Different curves correspond to different temperatures. The data at $100 \mathrm{~K}$ are shown by a continuous line in all cases: (a) carbon-carbon correlations term Icc (Q); (b) deuteron-deuteron correlations term I $\mathrm{DD}(\mathrm{Q})$; (c) carbon-deuteron correlations term $\mathrm{I}_{\mathrm{CD}}(\mathrm{Q})$. In parts $\mathrm{b}$ and c, the different curves corresponding to different temperatures can be identified from the bottom at $Q=1.25 \AA^{-1}$ : 100,314 , $363,413,463$, and $513 \mathrm{~K}$.

sample) as shown in Figure 3. The main feature of this temperature dependence is the intensity increase of the first peak of I(Q), which already starts at low temperatures bel ow the glass transition of PI. According to our previous discussion, at $100 \mathrm{~K}$ this first peak of I(Q) basically originates from the contribution to the scattering from the carbon-carbon and in particular from carbon chain-carbon chain correlations (see Figure 7b). Therefore, at first glance, we would expect that the intensity increase with temperature relates to these correlations. To investigate this question, we have calculated from the simulation results at different temperatures the evolution of the main contributions to the coherent scattering in the PId8 sample, i.e., $I_{C C}(Q)=\left\langle b_{C}\right\rangle^{2} A_{C C}(Q) ; I_{D D}(Q)=\left\langle b_{D}\right\rangle^{2} A_{H H}(Q) ; I_{C D}(Q)=$ $\left\langle b_{c}\right\rangle\left\langle b_{D}\right\rangle A_{C H}(Q)$. The incoherent contribution to the total intensity I(Q) shown in Figure 3 is only a constant term given by eq 5 (see section 3 ).

Figure 9 displays the temperature dependence of the three contributions $I_{C C}(Q), I_{D D}(Q)$, and $I_{C D}(Q)$. Apart from the shift with temperature toward lower Q 
values, the intensity of $I_{C c}(Q)$ increases only weakly from 100 to $314 \mathrm{~K}$, then remains more or less constant until $363 \mathrm{~K}$ and thereafter slightly decreases again toward high temperatures (see Figure 9a). We immediately realize that this behavior cannot explain the strong temperature increase of the first peak of I(Q) evident from Figure 3 . In the $Q$ region of about $1 \AA^{-1}$ where $(\mathrm{Q})$ has its first maximum, the contribution from deuteron-deuteron correlations, $\mathrm{I}_{\mathrm{DD}}(\mathrm{Q})$, also shows a slight intensity increase with temperature. Although this increase of intensity certainly contributes to the temperature behavior of the first peak of I(Q), again is not enough to explain this behavior. On the other hand, the contributions from the carbon-deuteron correlations (Figure 9c) display a dramatic change with temperature which manifests itself just in the $1-1.5 \AA^{-1} \mathrm{Q}$ region. In this region, $I_{C D}(Q)$ shows a minimum at $100 \mathrm{~K}$ which is close to zero in absolute value. This minimum transforms into a maximum al ready visible at $314 \mathrm{~K}$, which strongly develops with increasing temperature. Therefore, we may conclude that, although all atomic correlations in the PId8 sample contribute to the intensity increase of the first structure factor peak with temperature, this effect is mainly related to carbondeuteron correlations. Moreover, analyzing separately the different contributions to $I_{C D}(Q)$, we realize that the main contribution to the temperature evolution of $\mathrm{I}_{\mathrm{CD}}(\mathrm{Q})$ is due to both, carbon chain-deuteron chain and carbon chain-deuteron methyl correlations. On the contrary, carbon methyl-deuteron chain and carbon methyl-deuteron methyl correlations do not evolve significantly with temperature.

\section{Conclusions}

In this paper, we have shown that a combination of computer simulation and neutron diffraction experiments with polarization analysis on partially deuterated samples, is by now the best tool to unveil the atomic correlations contributing to the intermolecular structure of polymer melts and glasses. By measuring different partial structure factors of $\mathrm{PI}$, we have checked that our simulation cell is a realistic representation of the actual structure of this polymer. After that, taking advantage of the information contained in the simulation runs, we have unambiguously identified the different atomic correlations contributing to the different peaks of the total and partial structure factors measured. In particular, we found that the prepeak observed in the PId3 sample is not-as generally assumed-a sign of intermediate range order but evolves naturally from the combination and the weighting of different partial structure factors. We may speculate that this observation might have some consequences for the interpretation of the prepeaks in other complex materials. In addition, we have also shown-both by experimental and by simulations-that the intensity of the first intermolecular peak of the static structure factor strongly increases with temperature. Preliminary data from other polymers seem to indicate that this effect-which commences well bel ow the glass transition-could be a general feature of many amorphous polymers. Although a full understanding of this phenomenon will certainly need further work, we have been able to identify the main atomic correlations involved in this temperature evolution in the case of PI.

Acknowledgment. The authors acknowledge the Institute Laue Langevin (ILL, Grenoble, France) for experimental facilities. They also acknowledge Dr. Stewart and Dr. Hansen from the ILL for experimental support during neutron scattering measurements and Dr. Arbe from the CSIC (San Sebastián, Spain) for fruitful discussions. F.A. and J.C. also acknowledge the University of the Basque Country (Project 9/U PV00206.215-13568/2001), I berdrola S. A., and the Spanish Ministry of Science and Technology (Project MAT 2001/0070) for their support.

\section{References and Notes}

(1) Flory, P. J . Principles of Polymer Chemistry; Cornell University Press: London, 1953.

(2) Frick, B.; Richter, D.; Ritter, C. L. Europhys. Lett. 1989, 9 , 557.

(3) Zorn, R.; Richter, D.; Farago, B.; Frick, B.; Kremer, F.; Kirst U.; Fetters, L. Physica B 1992, 180\& 181, 534.

(4) Eilhard, J .; Zirkel, A.; Tschöp, W.; Hahn, O.; Kremer, K.; Schärpf, O.; Richter, D.; Buchenau, U. J . Chem. Phys. 1999, 110, 1819.

(5) Alvarez, F.; Alegría, A.; Colmenero, J .; Nicholson, T. M.; Davies, G. R. In CP479 Neutrons and Numerical MethodsN2M; J ohnson, M. R., Kearly, G. J ., Büttner, H. G., Eds.; American Institute of Physics: New York, 1999; p 201.

(6) Alvarez, F.; Arbe, A.; Colmenero, J . Chem. Phys. 2000, 261, 47.

(7) Alvarez, F.; Alegría, A.; Colmenero, J .; Nicholson, T. M.; Davies, G. R. Macromolecules 2000, 33, 8077.

(8) Colmenero, J .; Alvarez, F.; Arbe, A. Phys. Rev. E 2002, 65, 041804.

(9) Morton, M.; Fetters, L. J . Rubber Chem. Technol. 1975, 48, 359.

(10) Hadjichristidis, N.; I atrou, H.; Pispas, S.; Pitsikalis, M. J . Polym. Sci., Part A: Polym. Chem. 2000, 38, 3211.

(11) Placzek, G. Phys. Rev. 1952, 86, 377.

(12) Yarnell, J . L.; Katz, M. J .; Wenzel, R. G.; Koenig, S. H. Phys. Rev. A 1974, 7, 2130.

(13) Pusztai, L.; McGreevy, R. L. Physica B 1997, 234-236, 357; manuscript to be published.

(14) Zorn, R. Nucl. Instrum. Methods A 2002, 479, 568.

(15) Sun, H. J . Comput. Chem. 1994, 15, 752. Sun, H.; Mumby, S. J .; Maple, J. R.; Hagler, A. T. J . Am. Chem. Soc. 1994, 116 , 2978. Sun, H. Macromolecules 1995, 28, 701; 1994, 26, 5924. Sun, H.; Mumby, S. J .; Maple, J. R.; Hagler, A. T. J. Phys. Chem. 1995, 99, 5873; 1998, 102, 7338.

(16) See, for example: Sun, H. J. Phys. Chem. B 1998, 102, 7338.

(17) Theodorou, D. N.; Suter, U. W. Macromolecules 1985, 18, 1467; 1986, 19, 139; 1986, 19, 379.

(18) Mark, J . E., Ed. Physical Properties of Polymers Handbook; AIP Press: New York, 1996

(19) The structural relaxation time of our system, i.e., the relaxation time of the density-density correlation function at the $\mathrm{Q}$ value of the first intermolecular maximum of $\mathrm{S}(\mathrm{Q})$, is $\tau_{\alpha} \approx 300 \mathrm{ps}$. Therefore, the equilibration time used for the first MD run ( $1 \mathrm{~ns}$ ) is about 3 times $\tau_{\alpha}$. For the last run of $20 \mathrm{~ns}$, the system was in fact equilibrated during the equilibration time plus the time of the two previous MD runs, i.e., 4 ns in total. This time is about $12 \tau_{\alpha}$ (see ref 8 ).

(20) See, for example: Squires, G. L. Introduction of the Theory of Thermal Neutron Scattering; Dover: New York, 1996.

MA021397P 